\title{
Movilidad transnacional, trabajo y género: temporeras marroquíes en la agricultura onubense
}

\author{
Transnational mobility, agricultural work and gender: \\ morroccan female workers in huelva's agriculture
}

\author{
Juana Moreno NiEto \\ IESA-CSIC \\ jmoreno@iesa.csic.es
}

Recibido: 16.06 .2011

Aprobado definitivamente: 13.12.2011

\begin{abstract}
RESUMEN
Cada año miles de trabajadoras marroquíes de desplazan a los campos de agricultura intensiva de la provincia de Huelva, en el suroeste del estado español, para la plantación y/o recolección de la fresa. Se trata mayoritariamente de mujeres casadas y con hijos -acorde a las exigencias de estos programas- que permanecen allí entre tres y ocho meses cada año, con el compromiso de retornar a su país cuando finalice su contrato. En este artículo se presentan algunos resultados de una investigación que aborda estas migraciones, atendiendo a las interacciones y vínculos que tienen lugar entre los diferentes espacios recorridos por estas temporeras. Se analizarán, por un lado, los procesos que sostienen estas fórmulas de contratación, y por otro lado, la incidencia que este sistema de contrataciones tiene para sus protagonistas tanto a nivel material como simbólico, así como las estrategias desplegadas por ellas en el marco de las mismas.
\end{abstract}

Palabras clave: agricultura intensiva, género, Marruecos, temporeras, migraciones, contratos en origen.

\begin{abstract}
Thousands of Moroccan female workers move each year to the intensive agricultural fields of the province of Huelva -South West Spain- for the strawberry planting and/or harvesting. Most of them are married women with children, according to these programs demands. They stay between three and eight months each year, with the compromise of returning to their countries when their contracts are finished. In this review I will introduce some results drawn from a research that focuses on these migrations, specifically on the interactions and links that occur between the different spaces covered by the seasonal workers. On the one hand, I will analyze the processes that hold these contracting methods. On the other hand I will also focus on the impact these contracting methods have on these women at both material and symbolic level, just as the strategies they develop in this framework.
\end{abstract}

KEYWORDs: intensive agricultural, gender, Morocco, female seasonal workers, migrations. 


\section{SUMARIO}

1. Introducción. 2. El cultivo de la fresa y mercado de mano de obra en la agricultura onubense. 3. La incorporación de las temporeras marroquíes. 3.1. Perfil sociodemográfico de las temporeras marroquíes. 4. Contexto de origen: trabajo, familia y movilidad en Marruecos. 4.1. La salida: ruptura y negociación. 5. Trabajo y vida en las comarcas freseras. 5.1. Hábitat y movilidad. 6. La/s vuelta/s a Marruecos ¿Una vida entre dos lugares?. 6.1. La movilidad como recurso y "las pertenencias múltiples". 7. Algunas conclusiones.

\section{SUMMARY}

1. Introduction. 2. The cultivation of strawberry and labor market in agriculture of Huelva. 3. The incorporation of temporary Moroccan. 4. Context of origin: work, family and mobility in Morocco. 5. Working and living in the strawberry counties. 6. Returning to Morocco: A life between two places? 7. Some conclusions 


\section{INTRODUCCIÓN}

La contratación en origen de trabajadoras extranjeras ha constituido durante la primera década de este siglo la principal vía suministradora de mano de obra para la agricultura del sur de la provincia de Huelva (España). Así, entre 2007 y 2008 se llegaron a realizar casi 45.000 contratos en origen, en una campaña que moviliza alrededor de $60.000 \mathrm{em}-$ pleos. Este dato convierte, asimismo, a Huelva en la provincia con mayor número de contratos en origen para el sector agrario, acaparando el $62,5 \%$ de todas las contrataciones de esta modalidad realizadas en el Estado español en esos años y a gran distancia de la siguiente provincia, Lleida, que presentaba algo menos de 7.000.'

La fórmula de contratación en origen consiste en emplear braceras extranjeras que se desplazan con un contrato ya firmado, tras haber sido seleccionadas en su país de origen. Los contratos dan lugar a una autorización de trabajo y residencia de duración determinada (hasta un máximo de 9 meses al año), vinculada a una zona geográfica, a una ocupación y a un empleador concreto. Asimismo, una vez llegado a su término la trabajadora debe retornar a su país de origen para optar a ser llamada en posteriores campañas.

En el contexto de la agricultura onubense, estos contratos estuvieron dirigidos en un primer momento a mujeres de países de Europa del Este, si bien a partir de 2005 comenzaron a orientarse de manera significativa hacia trabajadoras marroquíes. El viraje hacia Marruecos por parte del empresariado agrícola se vio acompañado por la acción de las administraciones públicas españolas y marroquíes, así como por el apoyo financiero y técnico de la Unión Europea. De hecho, este programa de contrataciones en origen para la agricultura se ha visto erigido en modelo de gestión legal, ordenada y ética de los flujos migratorios a ambos lados del estrecho.
No obstante, una mirada atenta desvela cómo estas fórmulas de migración legal implican una rebaja de derechos para las trabajadoras, al tiempo que reproducen las asimetrías de género y desarrollo sobre las que se asientan.

El siguiente texto presenta algunos resultados de una investigación llevada a cabo entre los años 2008 y 2009 sobre las migraciones temporales de mujeres marroquíes en el marco de los programas de contratación en origen para el cultivo de la fresa en la provincia de Huelva². Se trata de una investigación de carácter cualitativo cuya metodología ha consistido fundamentalmente en la realización y posterior análisis de entrevistas en profundidad a los diferentes actores involucrados en estos programas, principalmente a las temporeras agrícolas. Asimismo, se analizaron un cierto número de datos secundarios que nos han permitido una aproximación más cuantitativa a determinados aspectos como el perfil socio-demográfico de las temporeras o el panorama del mercado laboral en el sector.

Con respecto a las entrevistas con las trabajadoras, la mayor parte de las mismas se efectuaron en la provincia de Huelva durante la campaña de recolección de la fresa de 2008 y fueron complementadas, más tarde, con otras realizadas en Marruecos con mujeres que habían participado en estos programas (2009). También fueron entrevistados otros actores relacionados con el sector de producción de fresas onubense y la gestión de la mano de obra agraria, fundamentalmente productores, responsables de organizaciones agrarias, sindicatos, e instituciones públicas, tanto en España como en Marruecos. Las entrevistas fueron realizadas en distintos idiomas: español, francés y marroquí. En el caso de las trabajadoras agrícolas, éstas se llevaron a cabo mayoritariamente en árabe marroquí, con el apoyo de una interprete nativa y, solo en algunos casos, en español $o$ en francés.

\footnotetext{
${ }^{1}$ Según datos del Ministerio de Trabajo y Asuntos Sociales de 2008, en 2007 se autorizaron 70.694 contrataciones en origen de temporada para la agricultura en el Estado español, de las cuales la provincia de Huelva acaparó 44.196 utilizados principalmente para la recolección de la fresa y, en menor medida, de otras frutas rojas y cítricos. A gran distancia, le seguían la provincia de Lleida con 6.867 de estos contratos (un 9,7\% del total nacional), la de Córdoba con 5.120 contratos (7\%), Almería con 4.088 contratos (5,8\%) y Segovia con 3.166 (4,5\%) (Gordo Márquez, 2008). Cabe señalar, no obstante, que a partir de la campaña 2009 el número de contratos en origen disminuirá sensiblemente en un contexto marcado por la recesión económica (véase nota 11).

${ }^{2}$ Se trata del proyecto de investigación realizado para la obtención del Diploma de Estudios Avanzados en la Universidad Autónoma de Madrid en 2009, en el marco de una beca de Formación de Profesorado Universitario (FPU). No obstante, en este texto se han incluido ciertos datos actualizados y originales producidos a posteriori.
} 
Partiendo de una concepción integradora de los flujos migratorios, se ha privilegiado un doble nivel de análisis. Un primer nivel, mesosociológico, que atiende a la arquitectura de los procesos que sostienen estas fórmulas de contratación, y un segundo nivel, microsociológico, centrado en las experiencias migratorias de sus protagonistas. En este marco, proponemos un enfoque que, de manera dinámica y superando la dicotomía sociedad de origen/sociedad de destino, preste atención a las interacciones y los vínculos que existen entre los diferentes espacios en los que se desarrolla la movilidad de estas temporeras. Semejante aproximación nos remite a la noción de "campo migratorio", que G. Simon define como "el conjunto del espacio estructurado por los flujos migratorios y relacionales, los espacios recorridos, practicados, vividos, por las poblaciones migrantes" (1981, cit. en Arab, 2008). Asimismo, entendemos que estos sistemas de contratación, así como las subjetividades y experiencias de las migrantes protagonistas, se ven atravesados por distintos sistemas de estratificación como el género, la clase o la nacionalidad, pudiendo cada una de estas dimensiones matizar, reforzar o desdibujar las otras. Estas tensiones y sinergias que se producen entre los distintos sistemas de estratificación constituyen, sin duda, un aspecto que intentaremos hacer emerger en nuestro análisis de los procesos de contratación en origen de temporeras marroquíes a lo largo de las siguientes páginas.

Para todo ello es preciso empezar por retratar la evolución de la gestión de la mano de obra en el contexto de la agricultura onubense a fin de entender la manera en que se produce la llegada de las temporeras de origen marroquí. Aunque los flujos de contratación en origen pudieran parecer a priori intercambiables de un país a otro, no se puede obviar que existen lógicas inherentes a las trayectorias de los distintos colectivos. De ahí que sea de suma importancia entender quiénes son estas trabajadoras, el contexto y los motivos de salida de las mismas, sus proyectos migratorios y las características de sus estancias en la provincia de Huelva. En defini- tiva, en este artículo, además de intentar contribuir a la comprensión de las lógicas contextuales y de la impronta de variables estructurales propias de un sector agrario intensivo insertado en los circuitos globales de comercialización, buscaremos desentrañar los significados que estas movilidades adquieren para sus protagonistas.

\section{EL CULTIVO DE LA FRESA Y MERCADO DE MANO DE OBRA EN LA AGRICULTURA ONUBENSE}

El arco litoral onubense se ha convertido, en pocas décadas, en la principal zona productora de fresas de Europa, ${ }^{3}$ y la segunda a nivel mundial, sólo por detrás de California, en los Estados Unidos. La disponibilidad de acuíferos subterráneos y unas buenas condiciones climatológicas, junto con la aplicación de elevadas cantidades de insumos agroquímicos para la preparación de la tierra y el tratamiento de las plantas, hacen que los campos onubenses constituyan hoy un enclave adecuado para el cultivo extra temprano de este cultivo. Estas circunstancias permiten introducir la producción en el mercado europeo hasta dos meses antes que los productores tradicionales como Italia o Francia, garantizando la competitividad de la misma. La exportación constituye, así, el destino mayoritario de la producción de fresa en Huelva, siendo los principales mercados importadores Alemania, Francia y Reino Unido (Aragón, 2006).

Se trata de una agricultura de carácter intensivo que requiere importantes inversiones en insumos (plantones, plásticos, productos fitosanitarios, riego, etc.) y una abundante mano de obra, especialmente para el periodo de recolección. La financiación de los insumos aplicados, junto al monopolio y control que sobre los precios detentan las grandes cadenas europeas de distribución, conllevan una importante presión económica sobre los productores. ${ }^{4}$ Así, si durante años se ha hablado del "oro rojo" en la provincia de Huelva, y siendo cierto que muchos em-

\footnotetext{
${ }^{3}$ Recientemente se han introducido otras variedades en la regióncomo la frambuesa, el arándano, los cítricos o los frutales. No obstante, la fresa es, sin duda, el cultivo mayoritario. En 2005 el cultivo de la fresa ocupaba un total de 6.692 hectáreas y la producción era de unas 289.998 toneladas (JDA, 2005).

${ }^{4}$ Los costes de producción aumentaron en un 70\% desde 1985 hasta 2005 . Por ello encontramos entre los productores unas altas tasas de endeudamiento (JDA, 2003).
} 
presarios agrícolas se han enriquecido rápidamente con el mismo, existe un consenso en que la rentabilidad de este cultivo disminuye cada año, ${ }^{5}$ al tiempo que gran parte de los beneficios se marchan fuera de la comarca. ${ }^{6}$

Ante esta situación las respuestas de los productores han ido fundamentalmente en dos direcciones complementarias: por un lado, incrementar la producción por hectárea, alimentando la insostenible espiral intensificadora, y por otro, reducir los costes de mano de obra. Con respecto a esta última, se ha apostado por el mantenimiento a la baja de la retribución salarial ${ }^{7} \mathrm{y}$ el aumento del rendimiento de trabajadores y trabajadoras mediante un control directo en los campos, especialmente en la recolección (Aragón, 2006). En este sentido, la presencia de trabajadores y, sobre todo, trabajadoras extranjeras, en sus diferentes regímenes, ha permitido, al igual que en otras zonas de agricultura intensiva (Santana, 1997), disponer de una mano de obra dispuesta a cubrir estos puestos de trabajo de baja remuneración. Hecho que ha derivado en la configuración de un mercado de trabajo con una acuciada segmentación étnica y sexual.

En las dos últimas décadas ha tenido lugar en Huelva una progresiva sustitución de la mano de obra autóctona por mano de obra inmigrante y femenina que no ha sido en absoluto aleatoria, sino que ha seguido una tendencia bien definida en base al establecimiento de un sistema jerarquizado de preferencias, que se asemeja a lo que se ha denominado para otros contextos un filtro étnico ${ }^{8}$ (López García, 2002). Se pueden identificar tres momentos de cambio en las tendencias de empleo en la agricultura onubense en la que tradicionalmente habían trabajado jornaleros y jornaleras procedentes de las provincias de Huelva, Cádiz y Sevilla, así como del sur de Portugal. Un primer momento, a finales de la década de los 90, con un incremento significativo del empleo de trabajadores inmigrantes magrebíes y subsaharianos. Un segundo momento, a partir del 2002, con la implementación de los contratos en origen y la llegada de las temporeras de países del Este de Europa. Cabe señalar que la sustitución de trabajadores magrebíes y subsaharianos por trabajadoras contratadas en origen tiene lugar en un contexto marcado por las malas campañas (JDA, 2003b) y por una creciente desconfianza hacia los trabajadores magrebíes dado, entre otros motivos, el protagonismo de este colectivo en las movilizaciones sociales que tuvieron lugar entre el 2000 y el 2002 (Gualda y Ruiz, 2004)9. Asimismo, a nivel nacional, el deterioro de las relaciones hispano-marroquíes entre 1999 y 2002 dificultaron las escasas contrataciones aún programadas en este país. ${ }^{10}$ Finalmente, un tercer momento de cambio se vislumbra a partir de 2006 con un retorno del protagonismo de Marruecos, esta vez de mujeres contratadas en origen, y la tímida incorporación de mano de obra procedente de países de otros continentes como Senegal o Filipinas (2008).

\footnotetext{
${ }^{5}$ La rentabilidad por hectárea de fresa (cociente entre costes de producción y beneficios por hectárea) ha descendido de un $23 \%$ a principios de los años 80 a un 5,5\% a principios del siglo XXI.

${ }^{6}$ Hasta un $73 \%$ del valor añadido que genera la venta de la fresa en destino queda en manos ajenas a los productores onubenses (Aragón, 2006).

${ }^{7}$ Huelva, junto con Almería, presenta la remuneración salarial más baja del Estado español (García y Ocaña, 2006). Aun así, desde las asociaciones de productores se denuncia que el aumento del jornal habido hasta ahora no permite garantizar la viabilidad del cultivo a medio plazo (Verdier, 2007). Según la Revisión Salarial de 2007 del Convenio Colectivo del Campo para la Provincia de Huelva de 2005, el salario sería de 35,66 euros/día para la plantación y 34,20 euros/día para la recolección y otras tareas (Boletín Oficial de la Provincia ${ }^{\circ}$ 58). En 2008 se firma un nuevo Convenio Colectivo del Campo en la provincia y en base a la revisión salarial de 2009 (BOPH n ${ }^{\circ}$ 98) este asciende a 38,91 euros/días para la plantación y 37,32 euros/día para la recolección y otras tareas.

${ }^{8} \mathrm{El}$ autor utiliza este término para hacer referencia a la voluntad política expresa de favorecer la entrada de aquellos inmigrantes considerados "más afines a nosotros" por cultura o religión, como aquellos de Latino América o Europa del Este (para un breve análisis de esta situación en el la política migratoria español entre el 2000 y el 2004véase también Izquierdo, 2004). No obstante, en el contexto onubense, la preferencia por unas nacionalidades sobre otras consideramos que se debe a un complejo entramado en el que entran en juego estereotipos y prejuicios de orden culturalista pero también dinámicas relacionadas con el mercado laboral autóctono.

${ }^{9}$ Durante esos años tuvieron lugar diferentes acciones reivindicativas contra la ley de extranjería, así como diversas movilizaciones por las precarias condiciones de la mano de obra extranjera en la agricultura de la provincia. En 2000/2001 hubo cinco encierros en la ciudad de Huelva protagonizados principalmente por ciudadanos marroquíes.

${ }^{10}$ A este respecto véase, por ejemplo, El País, 11 noviembre 2002.
} 
Las contrataciones en origen de trabajadoras de sexo femenino se afianzan, por tanto, como fórmula preferencial para el empleo agrícola en la zona durante gran parte de la década. ${ }^{11}$

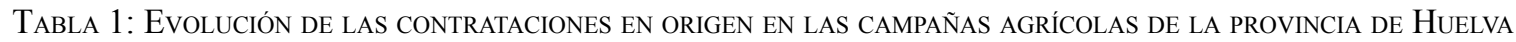

\begin{tabular}{|c|c|c|c|c|c|c|c|c|c|c|}
\hline & Bulgaria & Colombia & Ecuador & Filipinas & Marruecos & Polonia & Rumania & S & Ucrania & Total \\
\hline $\begin{array}{c}1999 / \\
2000\end{array}$ & & & & & & 600 & & & & 600 \\
\hline $\begin{array}{c}2000 / \\
2001\end{array}$ & & & & & 198 & 540 & & & & 738 \\
\hline $\begin{array}{c}2001 / \\
2002\end{array}$ & & 149 & & & 336 & 4954 & 970 & & & 6409 \\
\hline $\begin{array}{c}2002 / \\
2003\end{array}$ & & 177 & 15 & & 95 & 7535 & 4178 & & & 12000 \\
\hline $\begin{array}{c}2003 / \\
2004\end{array}$ & 508 & 105 & 8 & & 620 & 8506 & 10589 & & & 20336 \\
\hline $\begin{array}{c}2004 / \\
2005\end{array}$ & 604 & 82 & 64 & & 1094 & 7361 & 13186 & & & 22391 \\
\hline $\begin{array}{c}2005 / \\
2006\end{array}$ & 941 & 8 & 26 & & 2330 & 9796 & 19153 & & & 32254 \\
\hline $\begin{array}{c}2006 / \\
2007\end{array}$ & 3021 & 22 & 12 & & 5277 & & 20710 & & & 29042 \\
\hline $\begin{array}{c}2007 / \\
2008\end{array}$ & 4656 & 11 & 14 & 270 & 13600 & & 20634 & 749 & 557 & 40491 \\
\hline $\begin{array}{c}2008 / \\
2009 *\end{array}$ & 373 & 0 & 7 & 0 & $10416 * *$ & 0 & 3743 & 40 & 183 & 14722 \\
\hline $\begin{array}{c}2009 / \\
2010\end{array}$ & 0 & 0 & 0 & 0 & $4573 * * *$ & 0 & 0 & 0 & 0 & 4573 \\
\hline
\end{tabular}

${ }^{11}$ El contexto de crisis económica y malas cosechas de las campañas de 2008/2009 y 2009/2010 ha hecho descender el número de contratos en origen, especialmente en la campaña 2009/2010 (fueron 4.573 y solo en Marruecos). Cabe decir que ello se debe más a una imposición política que a un deseo de la patronal de abandonar estas fórmulas de contratación. De hecho se observa cómo las organizaciones agrarias siguen organizando viajes de temporeras desde Rumania y Bulgaria, que han dejado de ser contabilizadas como contratadas en origen tras el levantamiento de la moratoria para la libre circulación de trabajadores y trabajadoras en 2009. Por otra parte, la aprobación en junio 2010 del proyecto europeo M@res, "Sistema para la Movilidad de Flujos Migratorios Laborales en la Provincia de Huelva", financiado por la UE (1,4 millones de euros), para la consolidación y afianzamiento del flujo migratorio entre Marruecos y España, que da continuidad al proyecto AENEAS Cartaya, da cuenta de la voluntad existente de no abandonar estas fórmulas de contratación. 


\section{LA INCORPORACIÓN DE LAS TEMPORERAS MARROQUÍES}

Desde el año 2005, el número de contratos en origen realizados en Marruecos aumentará de manera significativa. Así, a pesar de los prejuicios existentes en la zona sobre los trabajadores marroquíes, ${ }^{12}$ una parte importante del empresariado agrícola se muestra de nuevo dispuesto a contratar nacionales de este país, siempre y cuando sean mujeres. Como en el caso de las temporeras el Este de Europa (Reigada, 2006), los discursos de los productores, organizacio- nes agrarias e instituciones sobre la preferencia por las trabajadoras de sexo femenino aluden a razones como que éstas "dan menos problemas", "son más trabajadoras" y "tienen más posibilidades de retorno" - en especial, si poseen cargas familiares - que sus homólogos varones. También se alude a capacidades que identifican como esencialmente femeninascomo la delicadeza o la sensibilidad en las manos que hace a las mujeres más válidas para la manipulación de la fresa. Finalmente, se identifica a las trabajadoras musulmanas con actitudes consideradas ventajosas para el trabajo, como que "no salen, no beben, etc.". ${ }^{13}$

TABla 2: Contrataciones EN ORIGEN DE MARRoQuíES

\begin{tabular}{|c|c|}
\hline $\mathbf{2 0 0 0 - 2 0 0 1}$ & 198 \\
\hline $\mathbf{2 0 0 1 - 2 0 0 2}$ & 336 \\
\hline $\mathbf{2 0 0 2 - 2 0 0 3}$ & 95 \\
\hline $\mathbf{2 0 0 3 - 2 0 0 4}$ & 620 \\
\hline $\mathbf{2 0 0 4 - 2 0 0 5}$ & 1094 \\
\hline $\mathbf{2 0 0 5 - 2 0 0 6}$ & 2330 \\
\hline $\mathbf{2 0 0 6 - 2 0 0 7}$ & 5277 \\
\hline $\mathbf{2 0 0 7 - 2 0 0 8}$ & 13600 \\
\hline $\mathbf{2 0 0 8 - 2 0 0 9}$ & $10416^{*}$ \\
\hline $\mathbf{2 0 0 9 - 2 0 1 0}$ & $4573^{* *}$ \\
\hline
\end{tabular}

${ }^{12}$ Las organizaciones agrarias y responsables locales nos relatan cómo los prejuicios sobre los trabajadores de nacionalidad marroquí existentes entre los productores han supuesto un freno a la contratación masiva de personas marroquíes en el sector. Con respecto a las percepciones sobre los diferentes grupos de personas extranjeras existentes en las comarcas freseras véase el Estudio sobre la exclusión social en la provincia de Huelva 2002 del Grupo de Estudios Sociales e Intervención Social de la Universidad de Huelva.

${ }^{13}$ Entrevistas a responsables del proyecto Aeneas-Cartaya, responsables de las organizaciones agrarias y empresarios agrícolas. Mayo 2008. Véase asimismo a este respecto "Dinar Ahadija y otras mil aguardan en la cola" EL País 18/01/2009. 
Entre las principales razones de este giro dado hacia Marruecos, señalar que la entrada en la Unión Europea primero de Polonia (2004) y más tarde de Rumania y Bulgaria (2007, con la definitiva liberalización de la circulación de trabajadores y trabajadoras en 2009), hasta la fecha principales países proveedores de mano de obra temporera en la zona, hacen temer al empresariado local un desplazamiento de estas trabajadoras hacia otros destinos europeos. No obstante, no puede obviarse el papel fundamental que va a jugar la voluntad política de ciertas administraciones locales, que en un contexto de buenas relaciones entre ambos países contará con el apoyo activo del gobierno central, en la elección del país magrebí como proveedor de trabajadoras para el sector.

Así, si bien ya existían experiencias particulares de empresarios que, instalados también en el país vecino, tramitaban de manera autónoma el reclutamiento, es en 2005 cuando tiene lugar la experiencia piloto que permitirá que al año siguiente se firme un acuerdo entre la municipalidad de Cartaya (Huelva) y la Agencia Nacional de Empleo y las Competencias de Marruecos (ANAPEC) para la gestión coordinada de estas contrataciones. Junto a las organizaciones agrarias, de carácter privado, estas dos instituciones públicas, con el apoyo técnico y financiero de la Unión Europea ${ }^{14}$ (y en su defecto del gobierno central) constituyen el verdadero motor que ha impulsado la contratación de temporeras marroquíes. Por su parte, la implicación activa de los representantes del Ministerio del Interior marroquí, sobre todo en las primeras fases de la inscripción de las trabajadoras, denota la importancia otorgada también por el reino alauita a estas contrataciones. Este carácter marcadamente institucional, en un proceso que en principio debía correr a cargo de las organizaciones de productores agrarios, supone un cambio con respecto a la naturaleza de las experiencias anteriores que se habían dado entre ambos países, y explica el reconocimiento público que reciben estas contrataciones, erigidas como ejemplo de ordenación de flujos migratorios legales entre ambas orillas del Mediterráneo e incluso como acción de lucha contra la pobreza en Marruecos ${ }^{15}$.

La mayor parte de las contrataciones que han tenido lugar hasta la fecha son de carácter genérico, es decir, se procede a la selección de candidatas en base a unos criterios determinados. Además de ser mujer, se han ido estableciendo requisitos relacionados con el origen y la vida familiar y reproductiva de las temporeras. En concreto, hasta ahora se ha exigido a las candidatas ser de procedencia rural, tener experiencia previa en la agricultura, menos de cuarenta y cinco años, estar en buen estado físico, y, desde la campaña 2006/2007, tener hijos menores a cargo y estar casadas, divorciadas o viudas. Quedando, por tanto, excluidas las madres solteras.

El establecimiento de estos criterios responde a la búsqueda de una mano de obra poco conflictiva, que presente un alto rendimiento en el trabajo y retorne a su país al finalizar la campaña. Con este fin, tanto el Estado español como el empresariado agrícola no dudan en desplegar estrategias basadas en lógicas sexistas que atentan contra la igualdad entre hombres y mujeres, las cuales se tornan aparentemente legítimas cuando de personas extranjeras se trata.

\subsection{Perfil sociodemográfico de las tempore- ras marroquíes}

En consonancia con los requisitos establecidos, y tomando como muestra los datos de la campaña 2007/2008, se observa que, efectivamente, el $99,4 \%$ de las personas marroquíes contratadas en origen que llegaron a los campos freseros son mujeres, de las cuales, 10.287 fueron primo-contratos y 1.931 repetidoras $^{16}$. Si tenemos en cuenta que en la campaña 2006/2007 se contrató a 5.277 trabajadoras, se deduce que han repetido menos de la mi-

\footnotetext{
${ }^{14}$ Entre 2005 y 2008, el programa trianual AENEAS concedido al ayuntamiento de Cartaya (Huelva) (2.000.000 de euros) y el programa MEDA II concedido a la ANAPEC (Marruecos) materializan la apuesta de la UE por la contratación de trabajadores con fecha de retorno. Este conocerá su continuación en junio de 2010 a través del programa M@res (Ver nota 11).

${ }^{15}$ Así, por ejemplo, la repartición de cuotas por regiones se apoya, entre otros, en el Mapa de la Pobreza marroquí elaborado por el Haut Comissariat au Plan en 2004. También se han incluido los 360 municipios rurales reconocidos por la Iniciativa Nacional para el Desarrollo Humano lanzada por el rey Mohamed VI en 2005. Sobre esta cuestión véase Desrues (2007).

${ }^{16}$ Dato: Aeneas-Cartaya. La elección de este dato, y no del proporcionado por la subdelegación el gobierno, se debe a que es el que constituye el total de la base de datos de la que extraeremos ciertas características sociodemográficas de las trabajadoras marroquíes.
} 
tad. Esta tendencia parece repetirse en la campaña 2008/2009, que frente a las previsiones al alza que hablaban de más $16.000^{17}$ contratos en Marruecos, finalmente contó con 10.416 trabajadoras marroquíes de las cuales al menos 4.101 fueron repetidoras. En cuanto al sexo, la excepción a la regla la conforman los 75 hombres contratados, probablemente conocidos de los empleadores o recomendados por otras trabajadoras. Por ello, queda justificado referirnos a las trabajadoras marroquíes en femenino y asimilar a ellas los datos sociodemográficos disponibles.

La mayor parte de estas temporeras tienen entre 31 y 40 años $(60,11 \%)$. Aproximadamente la mitad de ellas están casadas $(52,4 \%)$, mientras que un tercio de las mismas están divorciadas $(33,7 \%)$, un $11 \%$ son viudas $\mathrm{y}$, tan solo, un $1,5 \%$ son solteras. La presencia de mujeres de origen rural y casadas constituye una novedad con respecto a la tenden- cia observada en la emigración femenina marroquí hacia España, ya que hasta la fecha ésta se había identificado con mujeres de procedencia urbana y, principalmente carentes de vínculo marital (solteras, viudas y divorciadas) (Ramírez, 1999 y 2004).

Finalmente, señalar que, desde la campaña $2006 / 2007$, las temporeras provienen de puntos repartidos por casi toda la geografía del país vecino. Atendiendo a su distribución por regiones, en la campaña 2008, la Región del Gharb-Charda-Beni Hassan era la principal región de origen de las temporeras concentrando el $14,20 \%$ de las mismas Le siguen El Oriental (9,5\%), la región de Rabat-SaleZemmur-Zaer (9,2\%), Mequínez-Tafilalet $(8,8 \%)$ y Souss Massa-Draa (8,2\%). En total, la mitad de las temporeras contratadas $(49,9 \%)$ proviene de estas cinco regiones, todas caracterizadas por la presencia de un sector agrícola importante.

MaPA 1: Regiones DE ORIGEN DE LAS TEMPORERAS EN LA CAMPAÑA 2007/2008

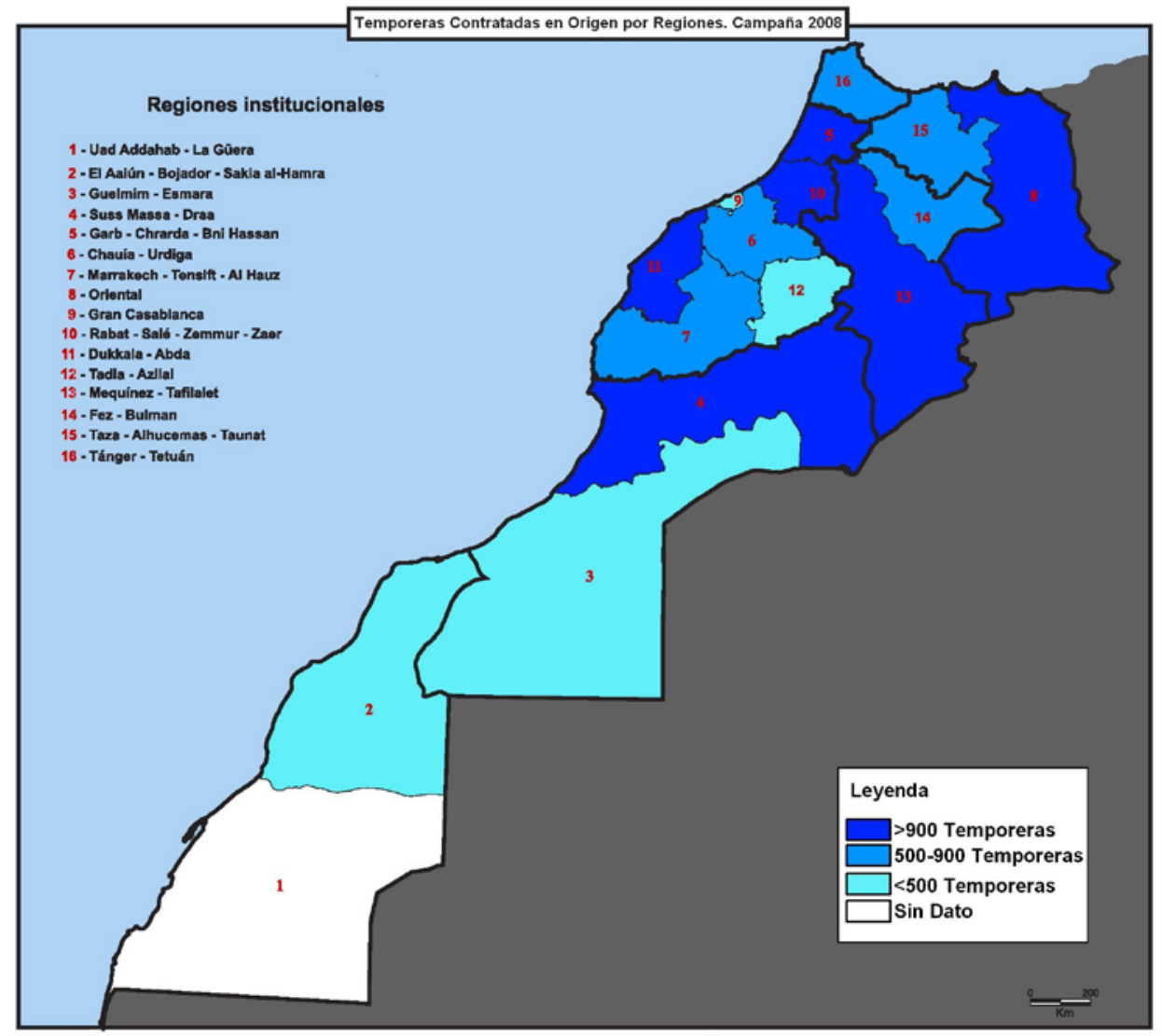

${ }^{17}$ Dato recogido por la subdelegación del gobierno de Huelva. Ver tablas. 


\section{CONTEXTO DE ORIGEN: TRABAJO, FAMILIA Y MOVILIDAD EN MARRUECOS}

Para contextualizar los significados que estas migraciones temporales tienen para sus protagonistas es fundamental conocer cuál era su posición en origen. Dos ámbitos concretos de sus vidas antes de emprender la migración han retenido nuestra atención: la familia y las ocupaciones. El análisis realizado ha permitido la identificación de dos perfiles básicos mujeres, las mantenedoras del hogar, es decir, aquellas que, solas o acompañadas, son las principales fuentes de sustento económico en sus familias ya en origen, y las amas de casa o ayudas familiares.

Entre las mujeres que han participado en nuestra investigación, la mitad realizaba algún tipo de trabajo remunerado en Marruecos justo antes de emigrar. Generalmente en empleos eventuales y muy precarios como asistentas domésticas, obreras en la industria agroalimentaria, o, sobre todo, jornaleras en la agricultura. Muchas trabajaban de manera estacional en los distintos cultivos de su región a cambio de un jornal que han situado entre los 30 y 50 dirhams al día. Son mujeres que dada su situación familiar (la mayoría pertenecen a familias en las que el hombre está ausente o fuera del mercado laboral) constituyen la fuente principal de ingresos de la familia, cuando no la única y, por ello, las hemos denominado mujeres mantenedoras del hogar. Para todas, la contratación en origen ha supuesto una continuidad en sus actividades laborales con una mejora en sus condiciones salariales. Existe, no obstante, un perfil de mujeres que se diferenciaría del resto por su mayor nivel de estudios y el tipo de empleo que desarrollaban en Marruecos. Ocupaban empleos mejor pagados y mejor considerados que los anteriores, en la industria o los servicios. El paso al trabajo agrícola constituye para ellas una pérdida del estatus de las actividades que realizan así como un incremento de la penosidad de las tareas. No obstante, la posibilidad de salir al extranjero y la promesa salarial compensarían, en principio, esta situación.

Un segundo grupo lo conformarían las entrevistadas que no trabajaban fuera del hogar en el momento anterior a emigrar a Huelva. Son, en su mayoría, mujeres casadas, aunque también hay viudas y solteras. Bajo su punto de vista el trabajo femenino fuera del hogar en Marruecos trae consigo una mala reputación para las mujeres (hchûma) ${ }^{18}$ ya que se relaciona con actividades deshonrosas o poco correctas. $\mathrm{Su}$ paso al empleo remunerado en la emigración queda para ellas justificado gracias al mayor estatus atribuido al trabajo en el extranjero en la sociedad marroquí (Ramírez 1999; IOE 2000). Por ello, el extranjero se les presenta como una oportunidad para el acceso al empleo remunerado de un modo legítimo.

\subsection{La salida: ruptura y negociación}

Todas las mujeres de este estudio se embarcaron en el proceso de contratación por iniciativa propia, si bien la mayoría debió negociar su salida en el seno de sus hogares. Migran, finalmente, con el permiso la autoridad familiar - cuando existe- y con el apoyo económico de otros familiares. ${ }^{19} \mathrm{La}$ estacionalidad de estas migraciones, así como el carácter institucional del proceso o el hecho de que a menudo sean numerosas las mujeres de un mismo pueblo que parten para España son factores que probablemente rebajen el carácter rupturista que la emigración femenina fuera del marco familiar, especialmente para las mujeres casadas, comporta para la ideología de género tradicional marroquí. No obstante, no lo elimina. De hecho, las entrevistadas nos relatan cómo, a menudo, están expuestas a críticas en sus comunidades de origen que cuestionan el objetivo real de su viaje o sus intenciones de retorno. Bajo el pretex-

\footnotetext{
${ }^{18}$ El concepto Hchuma (traducido habitualmente por vergüenza) nos lleva a hablar de moralidad y reputación en la sociedad marroquí lo que, por su parte, nos remite a la cuestión del control social ejercido sobre las mujeres. Señala Soumaya Naamane-Guessous (1991) que el término hchuma se refiere a "un código al cual uno se adhiere sin reflexionar y que legisla todas las situaciones de la existencia. Es el qué dirán que da miedo y el descrédito que caerá no solamente sobre el culpable, sino también sobre sus próximos y entorno" (traducción nuestra). López Lindstrom (2008) va más allá y se interesa asimismo por cómo este concepto es interiorizado por mujeres de origen marroquí modelando sus prácticas sociales.

${ }^{19}$ Todas las entrevistadas tuvieron que pedir dinero prestado para emprender la emigración. Tras cotejar las cifras ofrecidas por distintas fuentes y comprobar los gastos fijos de los diferentes procedimientos administrativos, 2500dh (unos 230 euros) sería una cifra aproximada del gasto mínimo por mujer. Si bien existen casos en que se ha gastado mucho más debido a las distancias a recorrer, los casos de corrupción funcionarial, el pago por las trabajadoras de gastos que no les corresponderían u otros.
} 
to de que la demanda sea en exclusiva de mano de obra femenina, la sospecha de la prostitución o de conductas libertinas se hace presente en los sectores más conservadores de la sociedad.

"La gente me decía, que tú te vas a quedar alli, tú ya no vuelves, y yo les decía que no, y que me llamen los años que me llamen, yo siempre vendré a trabajar y me volveré (...) Los del pueblo no piensan nada, sólo empezaron a hablar cuando ha pasado eso del periódico, ${ }^{20}$ los islamistas, seguro son los que han escrito este artículo, porque les da envidia que los hombres no fueran y que las mujeres sí, se preguntan por qué se llevan a las mujeres y no a los hombres, (y dicen que) seguro que se llevan a las mujeres porque se van a prostituir"'(Keltum, 25 años, casada) ${ }^{21}$

Las razones para iniciar este proyecto migratorio que las mujeres presentan son principalmente de orden económico. Esto tiene sentido dada la naturaleza de estas migraciones, de carácter temporal, vinculadas a una actividad laboral concreta y a un contrato de duración determinada. La carestía de la vida en Marruecos, la precariedad o la falta del empleo allí y el nivel salarial más elevado de España son los motivos más repetidos. No obstante, también aparecen otras causas de orden inmaterial o simbólico relacionadas con la imagen de éxito que rodea a la emigración a Europa o con procesos de realización personal de las migrantes (la reafirmación de su autonomía y de su capacidad resolutiva o el deseo de independencia con respecto la familia extensa, son algunas de ellas).

"No quiero venir a pasear...quiero venir a trabajar, a hacer lo que tengo que hacer y volver. (...) Dije que no, que quería venir a trabajar por mis hijos y quería mostrarme que soy valiente para trabajar fuera y para poder mantener a mis hijos". (Aicha, 37 años, viuda)
Ante las posibles críticas, muchas de las entrevistadas presentan sus motivaciones en la esfera de lo familiar. Es por trabajar para sus familias -señalan- que ellas están aquí. La afirmación de una de las mujeres entrevistadas cuando presentaba su experiencia en los campos onubenses es especialmente ilustrativa de esta realidad "yo no decidí ir a Huelva, simplemente que el trabajo estaba alli". Siendo para ellas evidente que el bienestar del grupo familiar es una razón contundente para emprender una emigración laboral de estas características, sin embargo, veremos que no es la única. Este discurso aparece de manera clara entre las mujeres casadas y con hijos, si bien se da de manera más atenuada, e incluso desaparece en algún caso, entre las solteras, aquellas que no tienen hijos o que poseen un mayor nivel de formación. De hecho, para un número importante de las participantes la contratación en origen constituye un medio para instalarse en Europa, algo que habían intentado realizar ya por otras vías. Esto es especialmente tangible en aquellas no presentes en este estudio que abandonaron los campos agrícolas antes de finalizar el contrato - las "fugadas" según se las denomina en el marco de los contratos en origen - pero también entre las que optan por permanecer en el programa a cambio de volver cada año, en ocasiones durante temporadas cada vez más largas, a la espera de llegar a ser regularizadas. ${ }^{22}$

"Sí, siempre lo he tenido de sueño (emigrar a Europa), pero la pobreza no me ha dejado. En verano del 2002 me iba a venir a Italia con un hombre, con los papeles de su mujer, pero la mujer me cogió celos y no vine. Además estaba muy ilusionada" (Hasania, 36 años, divorciada)

${ }^{20}$ El 10 de febrero de 2008, fue publicado en el periódico At-Tajdid, de tendencia ideológica próxima a los islamistas del Partido de la Justicia y el Desarrollo, el artículo "las mujeres rubias" en el que se acusaba a las mujeres marroquíes que fueron contratadas para la temporada de la fresa de costumbres morales poco recomendables e incluso de dedicación de parte de ellas a la prostitución. Agradezco a Thierry Desrues y Said Kirhlani el hecho de haberme proporcionado dicho artículo.

${ }^{21}$ Los nombres de las entrevistadas han sido sustituidos por otros ficticios a fin de preservar su anonimato.

${ }^{22}$ El artículo 40 de la LO 4/2000 señala que para la concesión de autorización de trabajo no se tendrá en cuenta la situación nacional de empleo cuando el contrato de trabajo vaya dirigido a las personas extranjeras que hayan sido titulares de autorizaciones de trabajo para actividades de temporada durante cuatro años naturales, periodo que ha sido reducido a 2 años en la redacción de la ley del 11 de diciembre 2009. No obstante, la dificultad de que este proceso de regularización se lleve a cabo estriba en que para ello es necesario un contrato anual que son minoritarios dado el carácter estacional de la agricultura de la región. Además, aunque esta posibilidad fue publicitada como una de las ventajas de la contratación en origen desde el proyecto AENEAS, y siendo cierto que 


\section{TRABAJO Y VIDA EN LAS COMARCAS FRESERAS}

El trabajo en la agricultura onubense conoce, junto con Almería, la remuneración más baja del Estado español y una fuerte exigencia de trabajo. No obstante, existe un importante diferencial salarial con el mundo agrícola marroquí ${ }^{23}$ que hace valiosos estos empleos para las temporeras de este país. Una vez en Huelva, la mayoría de las mujeres contratadas realizan labores de recolección, y algunas también de plantación, por un jornal de unos 35 euros brutos al día. ${ }^{24}$ En general, las temporeras entrevistadas se han mostrado cautelosas a la hora de valorar sus condiciones de alojamiento y trabajo. No obstante, no ha faltado un análisis crítico sobre las mismas que ha girado principalmente en torno a tres cuestiones: las condiciones de alojamiento, los gastos que han debido asumir irregularmente (material de trabajo, electricidad, viajes, etc. $)^{25}$ o la recurrente falta de trabajo una vez están en Huelva, ${ }^{26}$ problemas que en pocos casos habían denunciado ni comunicado a sus empleadores.

En efecto, un análisis de las condiciones legales y materiales que conforman las estancias de estas temporeras, da cuenta de cómo la valorada "poca conflictividad" atribuida a las mismas no es sino el resultado de unas condiciones construidas por este sistema de contratación transnacional. De hecho, cabe avanzar que esta docilidad se concibe abiertamente como un valor añadido ante los empleadores españoles, como demuestra alguna de las consignas dadas a las candidatas en las agencias de ANAPEC, aun en Marruecos. "(en ANAPEC) Me dijeron que aquí no nos iba a faltar de nada, que tenemos de todo, que no nos peleemos, que aquí iba a haber muchas nacionalidades, que callemos, que callemos, que nos callemos, que no protestemos por nada o que no critiquemos nada, que no hablemos y que no demos problemas, que ahi no nos va a hacer falta de nada, que tendremos de todo". (Khadiya, 40 años, casada)

Algunos de los factores que concurren en esta dirección son: en primer lugar, que dado el carácter estacional de sus estancias, los conocimientos de la realidad que les en la que se ven inmersas son muy incompletos: desconocen el convenio laboral al que están sujetas, los derechos que poseen en tanto que trabajadoras inmigrantes de temporada e incluso la mayoría no sabe en qué momento va a terminar su trabajo y ser devuelta a Marruecos. El desconocimiento del español aparece, también, como una importante traba a la hora de conectar con sindicatos y asociaciones en la zona que puedan reducir su desprotección..$^{27}$ En segundo lugar, debe tenerse en cuenta que estas mujeres provienen de contextos laborales bastante precarizados en Marruecos y, en general, poseen una escasa o nula cultura sindical. Por último, y sobre todo, estaría el temor a expresar descontento en un contexto en el que las posibilidades de retorno al año siguiente y el mantenimiento del permiso de residencia y trabajo dependen de la voluntad del empleador. ${ }^{28}$ Así, el estatus de los temporeros contratados en origen para la agricultura ha sido definido en otras latitudes como cautivo (Binford, 2006) en función de la cesión de libertades que estas perso-

se han realizado regularizaciones por esta vía, las consignas de la subdelegación del gobierno a fecha del 2010, en el contexto de recesión económica, advierten de la excepcionalidad de la posibilidad de utilizar esta vía de regularización. La subdelegación asume que, con carácter general, no existen trabajos de tal duración en las empresas agrícolas y se muestra claramente reticente a aprobar tales demandas, hecho que según las fuentes consultadas, se está materializando en el bloqueo de los expedientes solicitados.

${ }^{23}$ El Salario Mínimo Agrícola, SMAG, en Marruecos era de 52 DH en 2009 (unos cinco euros).

${ }^{24}$ Datos del 2007.

${ }^{25}$ El reglamento de la LOEXIX 4/2000 (RD 2393/2004) establece que los empleadores deberán sufragar los gastos de alojamiento de las temporeras así como el viaje bien de ida bien de vuelta, y el transporte semanal a los núcleos de población más cercanos para el abastecimiento en víveres.

${ }^{26}$ Los empresarios tienen la obligación de garantizar al menos 18 jornadas de trabajo a las temporeras que contratan.

${ }^{27}$ El programa Aeneas Cataya posee un equipo de mediadores que en la campaña 2007/2008 eran aproximadamente doce. Realizan tareas de traducción y mediación entre trabajadoras y empleadores si bien su actuación está lejos de ser asimilable a una labor sindical.

${ }^{28}$ Entrevistas realizadas a sindicatos y organizaciones sociales dan cuenta del escaso número de mujeres que acuden a ellas para denunciar incumplimientos del convenio y, que cuando acuden, son mayoritariamente casos en los que éstas ya han perdido su trabajo. Sobre las dificultades de cualquier tipo de movilización u organización sindical de este tipo de mano de obra véase Schmidt, 2005. 
nas realizan para trabajar legalmente en el país. Este calificativo se muestra apropiado para definir la condición de las temporeras marroquíes en los campos onubenses pues éstas ceden parte de sus libertades asociadas con un mercado de mano de obra libre (no tienen poder para negociar sus condiciones de trabajo, desplazarse en el mercado de trabajo o de elegir empleador). No obstante, no existe una ausencia física de libertad, pues se trata de una elección voluntaria y pueden abandonar en cualquier momento.

Por otra parte, este estatus queda reforzado por el hecho de que muchas de las temporeras residen en las explotaciones donde trabajan lo que dificulta una nítida separación entre la vida laboral y la vida privada de las mismas. En este contexto, los comportamientos de las trabajadoras en su tiempo libre se valoran a la hora de medir el potencial rendimiento del trabajo. Así, veíamos que los empleadores perciben como una ventaja el estereotipo existente sobre las temporeras marroquíes que, como buenas musulmanas, no salen mucho y no beben. Igualmente, cuando se producen incumplimientos en el contrato por parte de las trabajadoras, éstos se relacionan rápidamente con conductas "inmorales" como salir con hombres o ir de fiesta. ${ }^{29}$ Ello nos remite a la cuestión del control de la sexualidad y el cuerpo de las mujeres para el ejercicio y perpetuación de los mecanismos de dominación, en este caso para asegurar la disciplina de la fuerza de trabajo.

\subsection{Hábitat y movilidad}

Como decíamos, las temporeras residen normalmente en las fincas donde trabajan, alejadas de los núcleos de población..$^{30}$ El transporte mínimo obligatorio, , a proveer por el empleador para asegurar el desplazamiento hacia las localidades cercanas es de una vez por semana, si bien a veces se reduce a una vez a la quincena. Sin embargo, en todas las fincas en que se realizaron entrevistas las temporeras marroquíes iban más menudo al pueblo. Para ello se desplazaban en autostop, aprovechando los desplazamientos de capataces u otros trabajadores, en coches que ejercen como taxis ilegales en la zona o andando por la carretera.

Es en este contexto de acceso limitado a la movilidad donde debemos encuadrar los usos y discursos de las temporeras con respecto a sus relaciones con el entorno y desplazamientos. Así, observamos distintas actitudes: por un lado, estarían aquellas mujeres cuyas salidas se reducirían al mínimo imprescindible. Además de la falta de trasporte público y la distancia a los centros urbanos antes mencionada, el desconocimiento del idioma, la falta de costumbre de moverse en entornos desconocidos o la necesidad de ahorrar dinero son algunas de las razones avanzadas. Asimismo, encontramos en los discursos de algunas mujeres elementos que nos llevan a identificar mecanismos de preservación de una buena imagen, según la ideología de género tradicional marroquí, y eliminar cualquier resquicio de duda sobre la finalidad de su estancia.

"Nosotras hemos venido a trabajar, no a ponernos las mejores ropas. Estamos economizando al máximo. Todo lo que trabajamos, lo economizamos porque no queremos gastar ni un duro, porque todo el dinero lo necesitamos para gastar allí. Y no hay prostitutas ni nada. Cada una viene a lo que viene. Son mentiras todo. De Cartaya al Gato (referencia al albergue municipal). No nos movemos para nada, menos una vez que hemos ido a un mercadillo a la playa que nos dijeron que era barato y ya está. Pero que no vamos a ningún sitio”. (Hannan, 29 años, casada)

Las características de los permisos de trabajo y residencia, así como el modelo de alojamiento existente en la zona, ponen en tela de juicio el grado de autonomía del que disfrutan estas mujeres una vez salen de sus lugares de origen. Como hemos visto, emprenden el proyecto por iniciativa propia pero lo hacen bajo la tutela de una organización, primero, y de un empleador, después. Una de las entrevistadas ilustra muy bien esta situación e incluso utiliza este argumento para legitimar su migración.

\footnotetext{
${ }^{29}$ Esta identificación la encontramos en los discursos de empleadores, de los técnicos de las administraciones públicas y de las trabajadoras marroquíes (Entrevistas).

${ }^{30}$ Una excepción sería el albergue municipal de La "Casa del Gato" de titularidad pública. No obstante, éste reproduce algunas de las características del esquema residencial privado, es decir, está alejado de los núcleos de población, es de acceso restringido, existe una segmentación étnica y sexual del alojamiento, una limitación en el consumo de alcohol, etc.
} 
"No nos ha pasado nada. No hemos venido solas, han venido ellos a recogernos. Nos han acompañado hasta aquí. No nos ha pasado ningún problema. Hemos venido muy seguras y tranquilas. Es lo mismo porque llegamos aqui y nos encontramos con marroquies aqui, algunas conocidas (...) Quería demostrarme que soy valiente para venir aqui e igual que alli siempre he estado bajo el control y bajo el mando de mi padre, aquí podría estar perfectamente bajo el mando de un jefe". (Aicha, 37 años, viuda)

No obstante, ello no implica que no existan márgenes en el sistema dentro de los cuales las temporeras desarrollan diferentes acciones. Así, en contraposición, encontramos otras mujeres cuya movilidad es mucho mayor y que, más allá de las salidas pragmáticas para llamadas a sus familias y compras, declaran disfrutar de cierto ocio en el entorno. Algunas cuentan, incluso, con ciertas redes sociales en la comarca, principalmente familiares o amigas marroquíes. En nuestro estudio, las mujeres con mayores cuotas de movilidad y que disfrutan abiertamente de ellasllevan varias campañas viniendo, son solteras o divorciadas y menores de treinta años.

\section{LA/S VUELTA/S A MARRUECOS ¿UNA VIDA ENTRE DOS LUGARES?}

Los proyectos que estas trabajadoras poseen para $\mathrm{su} / \mathrm{s}$ vuelta/s a Marruecos son variados y dependen tanto de las características y la duración de su estancia en Huelva como de su situación familiar y laboral en sus comunidades de origen. A partir de los ingresos obtenidos y el uso que pretenden hacer de los mismos hemos distinguido seis proyectos tipo: 0. Proyectos nulos o fracasados. Sería el de aquellas temporeras que dada la corta duración de su estancia o los pocos días trabajados, apenas rentabilizarán la inversión realizada. Los escasos ingresos que obtengan los emplearán para cubrir gastos y realizar algún regalo a familiares. 1. Proyectos de sustento familiar, de aquellas mujeres mantenedoras del hogar que emplearán el dinero obtenido en seguir asegurando el sustento cotidiano de sus hijos y otros familiares y, en algunos casos, destinarán una parte para realizar alguna inversión extra como reformas de su vivienda. En este último caso, se trata de planes a largo plazo que pretenden sólo comenzar con los beneficios obtenidos en la presente campaña agrícola pues, dada la magnitud de los mismos, serían irrealizables con solo algunos meses de trabajo. 2. Proyectos de sustento familiar y ahorro personal, protagonizados por mujeres solteras que llevan trabajando varias campañas y permanecen en Huelva durante el periodo de plantación y recolección (casi ocho meses). Lo prolongado de sus estancias les permite desarrollar una importante capacidad de ahorro al tiempo que mantienen económicamente a sus familias en origen. 3. Proyectos complementarios a la economía familiar, de aquellas mujeres casadas que no trabajaban fuera del hogar en Marruecos y no pretenden sustituir el rol de "ganapan" de sus maridos. Conciben sus ganancias en Huelva como un aporte extra o complementario a la economía familiar para financiar algunas reformas en la vivienda, equipar la misma o sufragar determinados gastos como la educación de los niños. No pretenden incorporarse al mercado laboral a su retorno a Marruecos por lo que, en principio, consideran su trabajo en Huelva como una situación excepcional que no alteraría el tradicional reparto de roles en el seno familiar. 4. Proyectos de iniciación en el acceso a recursos, de aquellas viudas y divorciadas que han participado en la investigación que no trabajaban de manera remunerada en Marruecosy que pretenden hacerlo a la/s vuelta/s. La estancia en Huelva se les presenta, así, como una oportunidad de adquirir cierta autonomía económica para ellas y sus hijos con respecto a otros familiares. 5. Proyectos personales de aquellas mujeres cuyas familias cuentan ya con otros recursos. Estas mujeres, si bien invertirán en regalos para sus familiares, utilizarán los ingresos que obtengan en Huelva para su uso personal.

\subsection{La movilidad como recurso y "las perte- nencias múltiples"}

Queda puesto de manifiesto, pues, que muchas de las entrevistadas conciben sus estancias en Huelva de manera instrumental: trabajan allí a fin de mejorar sus condiciones y las de sus familias, en su comunidad de origen. Esto se enmarcaría en lo que ha venido a llamarse "la movilidad como recurso" (Morokvasik, 1999; Potot, 2005). Es evidente que existe una íntima interrelación entre ambos espacios el seno de sus experiencias migratorias. Así, pesar de sus ausencias, 
más o menos prolongadas del hogar, estas migrantes no abandonan su rol de cuidadoras y gestoras del hogar que materializan a través de recurrentes llamadas telefónicas y el envío periódico de dinero a las personas que se encargan de la manutención de sus hijos. Las tareas domésticas y el cuidado de los hijos son labores casi exclusivamente femeninas en Marruecos (Hafdane, 2004), por ello todas las entrevistadas han delegado estas funciones en otras mujeres de su familia para el cuidado de sus hijos, o también en vecinas o amigaspara las tareas de limpieza y cocina, conformando lo que se ha denominado las cadenas globales de cuidados (Hochschild, 2000).

Por otra parte, la duración de las estancias y los años que llevan desplazándose a Huelva, operan como factores clave a la hora de plantear la cuestión de las pertenencias múltiples. En el caso de algunas las repetidoras, especialmente entre aquellas cuya estancia alcanza los ocho meses de duración (para el periodo de siembra y recolección) ésta cuestión se hace muy patente. Tras tres o cuatro años participando en la campaña agrícola onubense, no conciben su vida sin estar en Huelva y asimilan sus estancias en Marruecos a un periodo vacacional. Aun así, se trata de mujeres mantenedoras del hogar que garantizan el sustento de sus familias en origen. Viven y trabajan aquí para mantener a sus familias allí, pero no exclusivamente. Estas mujeres desarrollan relaciones en las comarcas onubenses, principalmente con otras marroquíes. En nuestro estudio, todas son solteras o divorciadas y menores de treinta. Ninguna tiene relaciones de amistad con autóctonos, más allá de los jefes y algunos compañeros de trabajo y, en general, su conocimiento del idioma es limitado, si bien a través de la televisión y la radio algunas llegan a hablar un buen español.

"Llevamos ya 4 años juntas y nos llevamos bien, $y$ les enseñamos cosas a las nuevas. (entrevistadora: ¿echas de menos tú casa?)Muchísimo, llevamos 7 meses aquí. Nos quedamos aqui más que en Marruecos. En diciembre vamos unos 20 dias y volvemos. Me he acostumbrado a estar aqui, más que en Marruecos". (Najat, 24 años, soltera)
Malika, que suele venir una media de cuatro meses al año, desde hace tres, es un caso paradigmático de cómo su vida tanto laboral y socio-afectiva está enteramente dividida entre las provincias de Huelva (España) y Beni Mellal (Marruecos).

Lleva tres años viniendo, cuatro meses cada año. Durante el invierno se dedica a la confección en casa, formando parte de lo que sería un negocio familiar. Sus tíos y primos viven en Cartaya. Una vez a la semana se va a verlos y cuando no trabaja se queda allí a dormir. Le guardan las cosas de un año para otro. Su hermano, que vive desde hace años en Málaga, vino a verla un día esta temporada. Cuenta como el primer año le ofrecieron quedarse (sus tíos, su hermano...) pero ella decidió que prefería volverse que quedarse ilegal. Ahora tiene un novio, de origen marroquí, que vive en Lepe. Éste trabaja en agricultura y en la venta ambulante por las tardes. Los jueves o domingos viene a verla. Su familia lo conoce. Es de la misma zona que ella en Marruecos. Cuando está en Marruecos, ella habla por teléfono con la familia de él. Si se casara con él le gustaría quedarse en Huelva, mientras tanto prefiere ir y volver. (Malika, divorciada, 28 años) ${ }^{31}$

A pesar de todo ello, la estacionalidad de sus estancias y el grado, siempre existente, de incertidumbre a ser llamadas en las siguientes temporadas, impide cualquier planteamiento a largo plazo en el territorio español de manera regular, si bien su intención es de seguir viniendo en próximas campañas "mientras sea posible". Asimismo, la vinculación de sus permisos de residencia y trabajo a la actividad agrícola dificulta las posibilidades de movilidad social ascendente.

\section{AlgunAS CONCLUSIONES}

Empezábamos estas páginas postulando que el reconocimiento público del que se ha beneficiado este modelo de "migración circular" de temporeras agrícolas para el cultivo de la fresa ha contribuido a eclipsar las asimetrías de género y desarrollo so-

\footnotetext{
${ }^{31}$ Este fragmento, que no aparece en cursiva, procede de las notas tomadas en una de las entrevistas que no fueron grabadas en nuestra investigación.
} 
bre las que se asienta, al tiempo que invisibiliza los recortes en derechos fundamentales de las personas trabajadoras extranjeras que implica. En efecto, hemos podido comprobar cómo las condiciones legales y materiales que acompañan a los contratos en origen de temporada configuran una fuerza de trabajo que presenta altos niveles de desprotección, con escasa capacidad para negociar sus condiciones de trabajo.

La oferta de empleo dirigida exclusivamente a la población femenina y con cargas familiares procedentes de entornos de pobreza como el mundo rural marroquí, da cuenta de cómo los procesos de división internacional del trabajo del capitalismo globalizado se sustentan sobre las desigualdades de género, clase y nacionalidad existentes. En este caso, se aúnan los intereses del empresariado agrícola con la tendencia utilitarista de las políticas migratorias del estado español y la Unión europea que consiguen responder a la demanda de mano de obra de un determinado sector económico con unas trabajadoras que no lleguen a asentarse en el territorio.

Por otra parte, la aproximación a las experiencias migratorias de estas mujeres nos permite romper con el estereotipo de sumisión y pasividad, existente sobre las mujeres arabo-musulmanas en general, y rurales, en particular. En un contexto de demanda organizada de mano de obra, descubrimos mujeres mantenedoras del hogar que emprenden el periplo del traslado a otro país para asegurar la supervivencia de sus familias; mujeres casadas que no trabajaban en Marruecos, pero toman la iniciativa de incorporarse a los procesos de selección y a una actividad laboral, negociando su marcha con el cabeza de familia; jóvenes con estudios que buscan mejorar su situación y la de sus familias mediante un trabajo penoso que no se corresponde con su formación, etc.

Como la migración femenina, especialmente de madres de familia, en la sociedad rural marroquí rompe con la norma social, estas mujeres matizan sus ausencias mediante el aporte de ingresos a la familia y la hiperextensión de sus funciones como cuidadoras. Lejos de buscar una ruptura con el contexto, todas las protagonistas de nuestro estudio negocian sus salidas y parten con el consentimiento familiar mientras que, en sus discursos, muchas no dudan en enfatizar el carácter exclusivamente laboral de sus estancias en el extranjero a fin de legitimar su movilidad.

Para gran parte de las mujeres de este estudio, este tipo de migraciones se inscriben en lo que sería un uso de "la movilidad como recurso", en el sentido de que las migrantes se desplazan para acumular recursos y mejorar sus condiciones de vida en sus comunidades de origen. De hecho, las características de sus estancias favorecen esta concepción. No obstante, los significados que estas migraciones adquieren para sus protagonistas son complejos y varían en función de sus situaciones familiares en origen, la modalidad y antigüedad de sus estancias en Huelva o su edad, entre otras. Así, una parte de estas trabajadoras pasa cada año varios meses en Huelva tejiendo su existencia entre ambos espacios. La doble e incierta pertenencia de aquellas que pasan repetidamente ocho meses al año en la provincia de Huelva da buena prueba de ello.

En suma, se observa cómo, obligadas por la modalidad de permisos de residencia y trabajo, las temporeras desarrollan sus experiencias vitales entre dos escenarios, gestionando sus recursos materiales $y$ afectivos de manera transnacional a fin de optimizar sus posiciones tanto a nivel material como simbólico. Nos parece, sin embargo, inexacto hablar de "doble presencia" pues su existencia en los campos onubenses se revela incompleta, falta de derechos $\mathrm{y}$, sobre todo, de integración en el tejido social y perspectivas de cualquier tipo de movilidad social. Se puede afirmar, por tanto, que esta modalidad de contrataciones instituye la transnacionalización de la precariedad, ofreciendo de manera reglada condiciones precarias a personas provenientes de contextos también precarizados en Marruecos. 


\section{BIBLIOGRAFÍA}

Aragón, M.A. (2006): "El "oro rojo" en las tierras de Huelva. La extracción y explotación es siempre todavía” en ¿Qué hace esa fresa en tu mesa! La situación de l@s trabajadores de la fresa en Huelva. Editorial Atrapasueños, Sevilla.

BINFORD, L. (2006): "Campos agrícolas, campos de poder: el Estado mexicano, los granjeros canadienses y los trabajadores temporales mexicanos" Migraciones Internacionales 3, 3:54-80.

Bourdieu, P. (1991): El sentido práctico. Taurus, Madrid.

CRIADO, M.J. (1997): "Historias de Vida: el valor del recuerdo, el poder de la palabra” Migraciones 1:73-120.

Catarino, C. y M. Morokvasic, M. (2005): «Femme, genre et migration» Revue Européenne des Migrations Internationales 21,1:7-27.

Colectivo IOÉ (2000): Mujeres migrantes en España. Aproximación a sus proyectos migratorios. http://www. monografias.com/trabajos32/mujeres-migrantes-espana-proyectosr5-migratorios/mujeres-migrantes-espanaproyectos-migratorios.shtml \#_Toc128392732.

Del Rio, S. y A. Pérez Orozco (2004): “Una visión feminista de la precariedad desde los cuidados”. IX Jornadas de economía crítica, Madrid http://www.ucm.es/info/ec/jec9/pdf/A05\%20-20P\%e9rez\%20Orozco,\%20Amaia\%20 y\%20Del\%20R\%edo,\%20Sira.pdf.

Desrues, T. (2007): «Entre Etat de droit et droit de l'Etat. La difficile émergence de l'espace publique au Maroc» L'Année du Maghreb 2005-2006.CNRS Editions.

Gordo MÁrquez, M. (2008): "La contratación en origen de rumanos para actividades agrícolas de temporada" Cuadernos de Geografía 84:237-262.

García, J. y L. OcAÑa (2006): "Inmigrantes y freseros en la era de la globalización: el SOC contra la esclavitud del siglo XXI" en;Qué hace esa fresa en tu mesa! La situación de l@s trabajadores de la fresa en Huelva. Editorial Atrapasueños, Sevilla.

GuALDA, E. (2002): "La exclusión social en Huelva: la inmigración en una década de cambios" Estudio sobre la exclusión social en la provincia de Huelva, Grupo de Estudios Sociales e Intervención Social de la Universidad de Huelva. http://inclusion.sigadel.com/img_sistemas/ estudio\%20exclusion $\% 20$ social $\% 20 \mathrm{en} \% 201 \mathrm{a} \% 20$ provincia $\% 20 \mathrm{de} \% 20$ Huelva.pdf.

Gualda, E. y M. Ruiz García (2004): "Migración femenina de Europa del este y mercado de trabajo agrícola en la provincia de Huelva, España" Migraciones Internacionales 2,4:36-65.

Hadfane, H.L. (2004): «Les femmes marocaines entre le travail salarié et le travail familial. Stratégies et modes opérationnels, absences des politiques sociales» en L Achy,. y al, Marché du travail et genre Maghreb-Europe, Brussels Economic Series, Ed. Dulbea Asbi.

Hochschild, A. (2000): "Global Care Chains and Emotional Surplus Value" en W. Hutton, y A.Giddens, (eds.) On the Edge: Living with Global Capitalism, Jonathan Cape, Londres.

IzQuIERDO, A. (2004): "Los preferidos frente a los extranjeros permanentes: la inmigración marroquí en los inicios del siglo XXI" en López García, B. y M. Berriane (dirs.) Atlas de la Inmigración marroqui en España 2004. TEIMUAM, Madrid.

JunTa de Andalucía (2003): Determinación de la deuda agraria del sector fresero en la campaña 2003/2003. Consejería de Agricultura y Pesca. http://www.juntadeandalucia.es/agriculturaypesca/portal/www/portal/com/bin/ portal/DGPAgraria /Estudios_Prospectiva/Estudios_Informes/caracterizacion_de_sectores_y_productos/cultivos_ horticolas_y_protegidos/fresa1/deuda_fresa_2002_03/deuda_fresa_huelva_02_03.pdf.

JunTA DE ANDALUCía (2003b): Determinación de la deuda agraria del sector fresero de Huelva en la campaña 2.002/2003. Consejería de Agricultura y Pesca. http://www.juntadeandalucia.es/agriculturaypesca/portal/www/portal/com/bin/ portal/D GPAgraria/Estudios_Prospectiva/Estudios_Informes/caracterizacion_de_sectores_y_productos/cultivos_ horticolas_y_protegidos/fresa1/deuda_fresa_2002_03/deuda_fresa_huelva_02_03.pdf.

JunTa De Andalucía (2005): Anuario de Estadísticas Agrarias y Pesqueras Año 2005 . Consejería de Agricultura y Pesca. http://www.juntadeandalucia.es/agriculturaypesca/portal/www/portal/com/bin/portal/DGPAgraria/Estadisticas/ estadisticasagrarias/anuario2005.pdf.

López GarCíA, B. (2002): "El Islam y la integración de la inmigración social" Cuadernos de Trabajo social 15:129-143.

López Lindstrom, A. (2008): Mujeres migrantes entre dos mundos. Influencia y poder en el matrimonio transnacional. Altafulla, FIMAM. 
Miedes, B. y Redondo, D. (2007): Trabajadoras extranjeras en los campos freseros: de la necesidad a la invisibilidad II Congreso de Economía Feminista. http://www.unizar.es/economiafeminista/comunicaciones/area_2_mercado_ trabajo/Blanca_MIEDES\%20_UGARTE_comunicacion.pdf.

MoroKvasic M. (1999): «La mobilité transnationale comme ressource: le cas des migrants de l'Europe de l'Est»Cultures et Conflits 32: 105-122.

Ротот, S. (2005): «La place des femmes dans les réseaux migrants roumains» Revue Européenne des Migrations Internationales 1: 243-253.

Ramírez, A. (1999): «La valeur du travail : l'insertion dans le marché du travail des immigrées marocaines en Espagne» Revue Européenne des Migrations Internationales_15, 2: 9-36.

Ramírez, A. (2004): «Las mujeres marroquíes en España a lo largo de los 90» en B. López García y M. Berriane (dirs.) Atlas de la Inmigración marroqui en España 2004. TEIM-UAM, Madrid.

ReIgAdA, A. (2006): "De/ Vueltas al Campo. El Trabajo de las Mujeres en una Agricultura Globalizada" en ;Qué hace esa fresa en tu mesa! La situación de l@s trabajadores de la fresa en Huelva. Editorial Atrapasueños, Sevilla.

SANTANA, A. (1997): "La importancia de la mano de obra extranjera en las campañas agrícolas" Cuadernos de Relaciones Laborales 11, Servicio Publicaciones UCM.

ScнмidT, V. (2005): «Temporary migrant workers: organizing and protection strategies by trade unions» en C. Kupthsch (ed.), Merchants of labour, International Institute for Labour Studies, Geneva.

NaAmane-Guessous, S. (1991): Au-delà de toute pudeur. Eddif, Casablanca.

Schaefer Davis, S. (1996): «Un potentiel limité. Etat, femmes maghrébines et développement» en R. Bourquía, M.Charrad y N.Gallagher Femmes, culture et société II. Femmes pouvoir et développement. Ed. Afrique Orient, Casablanca.

Verdier, M. (2007): "Reflexiones en torno a la situación actual del sector de la fresa" Anuario Agricultura familiar en España. Unión de Pequeños Agricultores. http://www.upa.es/anuario_2007/pag_141-145_verdier.pdf. 